\title{
FURTHER CONSTRUCTIONS OF NESTED GROUP DIVISIBLE DESIGNS
}

\author{
Ying Miao*, Sanpei Kageyama* and Xiaoping Duan* \\ Some methods of construction for nested group divisible designs are given. \\ Cyclic nested group divisible designs are further discussed. Some individual \\ plans are also tabulated with 4 new E-optimal designs.
}

\section{Introduction}

Nested group divisible (NGD) designs, which are 3-associate partially balanced incomplete block (PBIB) designs, were introduced by Roy (1953), and subsequently studied by Raghavarao (1960), Roy (1962), Hinkelmann and Kempthorne (1963), Bhagwandas, Sinha and Kageyama (1992), Duan and Kageyama (1993), etc. These designs are useful as 3-factor experiments, having balance as well as orthogonal factorial structure. In this paper, several methods of construction of such designs are provided by breaking up the groups or the blocks of given group divisible (GD) designs. Additionally, a new type of nested GD design, which we call a cyclic nested group divisible (cyclic NGD or CNGD) design, is discussed. Finally some individual plans are also tabulated. This tabulation includes 4 new E-optimal nested GD designs.

An NGD design $(\mathcal{X}, \mathscr{S}, \mathcal{G}, \mathcal{B})$ is an arrangement with $v$ treatments into $b$ blocks such that $v=|\mathcal{X}|, b=|\mathcal{B}|, p=|\mathcal{\&}|, m=|\mathcal{G}|$, and

(i) each treatment $x \in \mathcal{X}$ is replicated $r$ times;

(ii) each block $B \in \mathcal{B}$ contains $k(<v)$ treatments;

(iii) there exists an association scheme on $v=p m n$ treatments, which are divided into $p$ sets $S \in \mathscr{S}$ of $m$ groups $G \in \mathcal{G}$ of $n$ treatments each, such that

(a) any two treatments from the same set and the same group are first associates;

(b) any two treatments from the same set but different groups are second associates;

(c) any two treatments from different sets are third associates;

(iv) any two treatments which are $i$ th associates occur together in $\lambda_{i}$ blocks, $i=1,2,3$.

Let $n_{i}$ denote the number of treatments which are $i$ th associates of any treatment. Then $n_{1}=n-1, n_{2}=(m-1) n, n_{3}=(p-1) m n$.

The present association scheme can be displayed in a $p m \times n$ array as

Received December, 1995, Revised July, 1996. Accepted July, 1996

* Department of Mathematics, Faculty of Science, Hiroshima University, Higashi-Hiroshima 739, Japan 


$$
\left[\begin{array}{c}
A^{(1)} \\
A^{(2)} \\
\vdots \\
A^{(p)}
\end{array}\right] \text { with } \quad A^{(i)}=\left[\begin{array}{cccc}
a_{11}^{(i)} & a_{12}^{(i)} & \cdots & a_{1 n}^{(i)} \\
a_{21}^{(i)} & a_{22}^{(i)} & \cdots & a_{2 n}^{(i)} \\
\vdots & \vdots & & \vdots \\
a_{m 1}^{(i)} & a_{m 2}^{(i)} & \cdots & a_{m n}^{(i)}
\end{array}\right], \quad i=1,2, \cdots, p
$$

where $a_{s t}^{(i)}$ denotes the $t$ th treatment of the $s$ th group in the $i$ th set. This representation is helpful for the proof of the theorems given in Section 2.

We shall denote the usual group divisible (GD) design by $\mathrm{GD}(v, b, r, k ; m$, $\left.n ; \lambda_{1}, \lambda_{2}\right)$ and the present NGD design by $\operatorname{NGD}\left(v, b, r, k ; m, n, p ; \lambda_{1}, \lambda_{2}, \lambda_{3}\right)$. Note that the NGD design with $p=1$ is the same as a GD design.

In an NGD design, if $\lambda_{1}=\lambda_{2}$ or $\lambda_{2}=\lambda_{3}$, the design reduces to a GD design by combining the first and the second or the second and the third associate classes. When $\lambda_{1}=\lambda_{2}=\lambda_{3}$, the design reduces to a balanced incomplete block (BIB) design by combining all of the associate classes.

The following notations are here used: $\boldsymbol{1}_{s}$ is an $s$-dimensional column vector with all elements unity, $I_{s}$ is the identity matrix of order $s, J_{s \times t}$ is an $s \times$ $t$ matrix with all elements unity, $J_{s \times s}$ is specially denoted by $J_{s}, 0_{s \times t}$ denotes an $s \times t$ matrix with all elements zero, $A \otimes B$ denotes the Kronecker product of two matrices $A$ and $B, A^{\prime}$ denotes the transpose of $A$, and $Z_{s}=\{0,1, \cdots, s$ $-1\}(\bmod s)$.

\section{Breaking up groups of GD designs}

In this section, we describe several patterned methods of construction by breaking up groups of a GD design.

THEOREM 2.1. The existence of a GD(pmn, $\left.b^{\prime}, r^{\prime}, k^{\prime} ; p, m n ; \lambda_{1}^{\prime}, \lambda_{2}^{\prime}\right)$ and $a$ $G D\left(m n, b^{\prime \prime}, r^{\prime \prime}, k^{\prime} ; m, n ; \lambda_{1}^{\prime \prime}, \lambda_{2}^{\prime \prime}\right)$ implies the existence of an $N G D\left(p m n, b^{\prime}+p b^{\prime \prime}\right.$, $\left.r^{\prime}+r^{\prime \prime}, k^{\prime} ; m, n, p ; \lambda_{1}^{\prime}+\lambda_{1}^{\prime \prime}, \lambda_{1}^{\prime}+\lambda_{2}^{\prime \prime}, \lambda_{2}^{\prime}\right)$.

PROOF. Let $N_{1}$ be the $p m n \times b^{\prime}$ incidence matrix of the first GD design and $N_{2}$ be the $m n \times b^{\prime \prime}$ incidence matrix of the second one. Then it can be shown that

$$
N=\left[N_{1}: I_{p} \otimes N_{2}\right]
$$

is the incidence matrix of the required NGD design.

REMARK 2.1. If $\lambda_{2}^{\prime}=\lambda_{1}^{\prime}+\lambda_{2}^{\prime \prime}$, we obtain a $\mathrm{GD}\left(p m n, b^{\prime}+p b^{\prime \prime}, r^{\prime}+r^{\prime \prime}, k^{\prime} ; p m\right.$, $n ; \lambda_{1}^{\prime}+\lambda_{1}^{\prime \prime}, \lambda_{2}^{\prime}$ ), which generalizes Theorem 3.2 of Duan and Kageyama (1995).

THEOREM 2.2. The existence of a GD(pmn, $\left.b^{\prime}, r^{\prime}, k^{\prime} ; p, m n ; \lambda_{1}^{\prime}, \lambda_{2}^{\prime}\right)$ and $a$ $G D\left(m n, b^{\prime \prime}, r^{\prime \prime}, k^{\prime}-(p-1) m n ; m, n ; \lambda_{1}^{\prime \prime}, \lambda_{2}^{\prime \prime}\right)$ implies the existence of an NGD $\left(p m n, b^{\prime}+p b^{\prime \prime}, r^{\prime}+r^{\prime \prime}+(p-1) b^{\prime \prime}, k^{\prime} ; m, n, p ; \lambda_{1}^{\prime}+\lambda_{1}^{\prime \prime}+(p-1) b^{\prime \prime}, \lambda_{1}^{\prime}+\lambda_{2}^{\prime \prime}+(p-1) b^{\prime \prime}\right.$, $\left.\lambda_{2}^{\prime}+2 r^{\prime \prime}+(p-2) b^{\prime \prime}\right)$.

PROOF. Let $N_{1}$ be the $p m n \times b^{\prime}$ incidence matrix of the first GD design and $N_{2}$ be the $m n \times b^{\prime \prime}$ incidence matrix of the second one. Then it follows that

$$
N=\left[N_{1}: I_{p} \otimes N_{2}+\left(J_{p}-I_{p}\right) \otimes J_{m n \times b^{\prime \prime}}\right]
$$


is the incidence matrix of the required NGD design.

THEOREM 2.3. The existence of a GD(pmn, $\left.b^{\prime}, r^{\prime}, k^{\prime} ; p, m n ; \lambda_{1}^{\prime}, \lambda_{2}^{\prime}\right)$ and $a$ $G D\left(m n, b^{\prime \prime}, r^{\prime \prime}, k^{\prime}-m n ; m, n ; \lambda_{1}^{\prime \prime}, \lambda_{2}^{\prime \prime}\right)$ implies the existence of an $N G D\left(p m n, b^{\prime}\right.$ $+p(p-1) b^{\prime \prime}, r^{\prime}+(p-1) b^{\prime \prime}+(p-1) r^{\prime \prime}, k^{\prime} ; m, n, p ; \lambda_{1}^{\prime}+(p-1) b^{\prime \prime}+(p-1) \lambda_{1}^{\prime \prime}, \lambda_{1}^{\prime}+(p$ $\left.-1) b^{\prime \prime}+(p-1) \lambda_{2}^{\prime \prime}, \lambda_{2}^{\prime}+2 r^{\prime \prime}\right)$ for any positive integer $p \geq 3$.

PROOF. Let $N_{1}$ and $N_{2}$ be the $p m n \times b^{\prime}$ and $m n \times b^{\prime \prime}$ incidence matrices of the first and the second GD designs, respectively. It can then be shown that

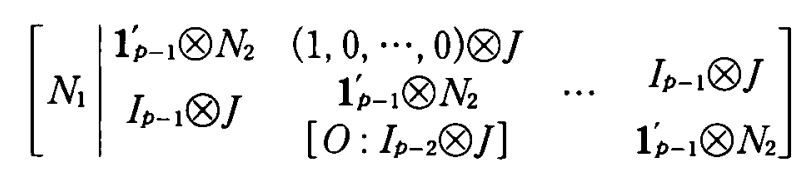

is the incidence matrix of the required NGD design.

A GD design with $v=2 k$ is said to be self-complementary. Note that a self-complementary design has the same block structure as its complement. Kageyama and Tanaka (1981) constructed several series of self-complementary GD designs. They can be applied to obtain the following observations. Let $\bar{N}$ $=J-N$.

THEOREM 2.4. The existence of a $G D\left(3 m n, b^{\prime}, r^{\prime}, k^{\prime} ; 3, m n ; \lambda_{1}^{\prime}, \lambda_{2}^{\prime}\right)$ and $a$ self-complementary $G D\left(m n, b^{\prime \prime}, r^{\prime \prime}, k^{\prime} / 3 ; m, n ; \lambda_{1}^{\prime \prime}, \lambda_{2}^{\prime \prime}\right)$ implies the existence of an $N G D\left(3 m n, b^{\prime}+4 b^{\prime \prime}, r^{\prime}+4 r^{\prime \prime}, k^{\prime} ; m, n, 3 ; \lambda_{1}^{\prime}+4 \lambda_{1}^{\prime \prime}, \lambda_{1}^{\prime}+4 \lambda_{2}^{\prime \prime}, \lambda_{2}^{\prime}+2 r^{\prime \prime}\right)$.

PROOF. Let $N_{1}$ and $N_{2}$ be the incidence matrices of the first and the second GD designs, respectively. Then it can be shown that

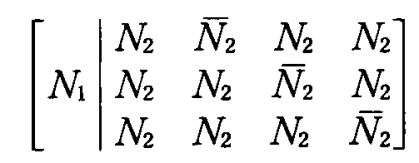

is the incidence matrix of the required NGD design.

THEOREM 2.5. The existence of $a G D\left(4 m n, b^{\prime}, r^{\prime}, k^{\prime} ; 4, m n ; \lambda_{1}^{\prime}, \lambda_{2}^{\prime}\right)$ and $a$ self-complementary $G D\left(m n, b^{\prime \prime}, r^{\prime \prime}, k^{\prime} / 4 ; m, n ; \lambda_{1}^{\prime \prime}, \lambda_{2}^{\prime \prime}\right)$ implies the existence of an $N G D\left(4 m n, b^{\prime}+4 b^{\prime \prime}, r^{\prime}+4 r^{\prime \prime}, k^{\prime} ; m, n, 4 ; \lambda_{1}^{\prime}+4 \lambda_{1}^{\prime \prime}, \lambda_{1}^{\prime}+4 \lambda_{2}^{\prime \prime}, \lambda_{2}^{\prime}+2 r^{\prime \prime}\right)$.

PROOF. The required design can be given by

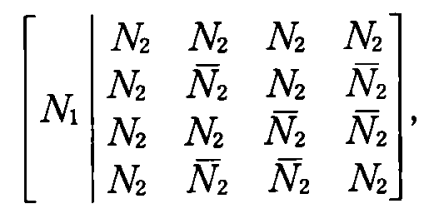

where $N_{1}$ and $N_{2}$ are the incidence matrices of the first and the second GD designs, respectively.

THEOREM 2.6. The existence of $a G D\left(3 m n, b^{\prime}, r^{\prime}, k^{\prime} ; 3, m n ; \lambda_{1}^{\prime}, \lambda_{2}^{\prime}\right)$ and $a$ self-complementary $G D\left(m n, b^{\prime \prime}, r^{\prime \prime}, k^{\prime} / 2 ; m, n ; \lambda_{1}^{\prime \prime}, \lambda_{2}^{\prime \prime}\right)$ implies the existence of an $N G D\left(3 m n, b^{\prime}+6 b^{\prime \prime}, r^{\prime}+4 r^{\prime \prime}, k^{\prime} ; m, n, 3 ; \lambda_{1}^{\prime}+4 \lambda_{1}^{\prime \prime}, \lambda_{1}^{\prime}+4 \lambda_{2}^{\prime \prime}, \lambda_{2}^{\prime}+r^{\prime \prime}\right)$. 
PROOF. Let $N_{1}$ and $N_{2}$ be the incidence matrices of the first and the second GD designs, respectively. The pattern as

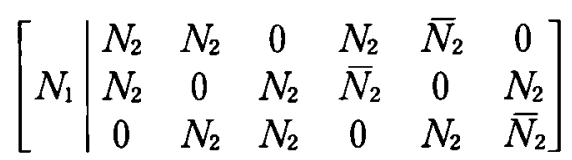

can yield the required NGD design.

THEOREM 2.7. The existence of a GD $\left(2 m n, b^{\prime}, r^{\prime}, k^{\prime} ; 2, m n ; \lambda_{1}^{\prime}, \lambda_{2}^{\prime}\right)$ and $a$ self-complementary $G D\left(m n, b^{\prime \prime}, r^{\prime \prime}, k^{\prime} / 2 ; m, n ; \lambda_{1}^{\prime \prime}, \lambda_{2}^{\prime \prime}\right)$ implies the existence of an $N G D\left(2 m n, b^{\prime}+2 b^{\prime \prime}+2 s, r^{\prime}+2 r^{\prime \prime}+s, k^{\prime} ; m, n, 2 ; \lambda_{1}^{\prime}+2 \lambda_{1}^{\prime \prime}+s, \lambda_{1}^{\prime}+2 \lambda_{2}^{\prime \prime}+s, \lambda_{2}^{\prime}+r^{\prime \prime}\right)$ for any positive integer $s$.

PROOF. The result follows from the pattern as

$$
\left[\begin{array}{c|cccc}
N_{1} & N_{2} & N_{2} & J_{m n \times s} & 0_{m n \times s} \\
N_{2} & \bar{N}_{2} & 0_{m n \times s} & J_{m n \times s}
\end{array}\right],
$$

where $N_{1}$ and $N_{2}$ are the incidence matrices of the first and the second GD designs, respectively.

Note that all of the constructions described above can be generalized by taking $t$ copies of the ingredient designs when breaking up the groups of the first GD design. Also note that the ideas in Bhagwandas, Sinha and Kageyama (1992) are used to break up the groups in some theorems.

\section{Breaking up blocks of GD designs}

The following construction is combinatorial in some sense.

THEOREM 3.1. The existence of a $G D\left(p m, b^{\prime}, r^{\prime}, k^{\prime} ; p, m ; \lambda_{1}^{\prime}, \lambda_{2}^{\prime}\right)$ and $a G D$ $\left(k^{\prime} n, b^{\prime \prime}, r^{\prime \prime}, k^{\prime \prime} ; k^{\prime}, n ; \lambda_{1}^{\prime \prime}, \lambda_{2}^{\prime \prime}\right)$ implies the existence of an $N G D\left(p m n, b^{\prime} b^{\prime \prime}, r^{\prime} r^{\prime \prime}\right.$, $\left.k^{\prime \prime} ; m, n, p ; r^{\prime} \lambda_{1}^{\prime \prime}, \lambda_{1}^{\prime} \lambda_{2}^{\prime \prime}, \lambda_{2}^{\prime} \lambda_{2}^{\prime \prime}\right)$.

PROOF. Let $(i, j), 1 \leq i \leq p, 1 \leq j \leq m$, be the treatment of the first GD design, where $(i, j)$ and $\left(i^{\prime}, j^{\prime}\right)$ are first associates if $i=i^{\prime}$ and $j \neq j^{\prime}$, and second associates if $i \neq i^{\prime}$. Now we define $n$ new treatments $(i, j, l), 1 \leq l \leq n$, for each old treatment $(i, j)$, and an NGD association scheme is stated as follows: $(i, j$, $l)$ and $\left(i^{\prime}, j^{\prime}, l^{\prime}\right)$ are first associates if $i=i^{\prime}, j=j^{\prime}$ and $l \neq l^{\prime}$, second associates if $i=i^{\prime}$ and $j \neq j^{\prime}$, and third associates if $i \neq i^{\prime}$. For each block $B^{\prime}=\left\{\left(i_{1}, j_{1}\right),\left(i_{2}, j_{2}\right)\right.$, $\left.\cdots,\left(i_{k^{\prime}}, j_{k^{\prime}}\right)\right\}$, we can construct a $\mathrm{GD}\left(k^{\prime} n, b^{\prime \prime}, r^{\prime \prime}, k^{\prime \prime} ; k^{\prime}, n ; \lambda_{1}^{\prime \prime}, \lambda_{2}^{\prime \prime}\right)$ on $B^{\prime} \times\{1,2, \cdots$, $n$ ) with block set $\mathcal{B}_{B^{\prime}}$. Then combining all of the $\mathcal{B}_{B^{\prime}}$ gives the block set of the required $\mathrm{PBIB}$ design based on the NGD association scheme.

This is a rather powerful, though simple method for the construction of NGD designs with small parameters, as shown in Section 5.

\section{Cyclic nested group divisible designs}

Now we deal with a special type of nested GD designs which we call a cyclic nested group divisible design. A construction method is given. This is 
based on the technique of Jimbo and Kuriki (1983) and Jimbo and Vanstone (1984).

Let $(\mathcal{X}, \mathscr{S}, \mathcal{G}, \mathcal{B})$ be an $\operatorname{NGD}\left(v, b, r, k ; m, n, p ; \lambda_{1}, \lambda_{2}, \lambda_{3}\right)$, and $\sigma$ be a permutation on $\mathcal{X}$. For any block $B=\left\{b_{1}, \cdots, b_{k}\right\} \in \mathcal{B}$, any group $G=\left\{x_{1}, \cdots, x_{n}\right\}$ $\in \mathcal{G}$ and any set $S=\left\{G_{1}, \cdots, G_{m}\right\} \in \mathscr{\&}$, define $B^{\sigma}=\left\{b_{1}^{\sigma}, \cdots, b_{k}^{\sigma}\right\}, G^{\sigma}=\left\{x_{1}^{\sigma}, \cdots, x_{n}^{\sigma}\right\}$ and $S^{\sigma}=\left\{G^{\sigma}, \cdots, G_{m}^{\sigma}\right\}$. If $\mathcal{B}^{\sigma}=\left\{B^{\sigma}: B \in \mathcal{B}\right\}=\mathcal{B}, \mathcal{S}^{\sigma}=\left\{G^{\sigma}: G \in \mathcal{G}\right\}=\mathcal{G}$ and $\mathscr{S}^{\sigma}$ $=\left\{S^{\sigma}: S \in \mathscr{S}\right\}=\mathscr{\&}$, then $\sigma$ is called an automorphism of $(\mathcal{X}, \mathscr{S}, \mathcal{G}, \mathcal{B})$. If there is an automorphism $\sigma$ of order $v=p m n=|\mathcal{X}|$, then the $\operatorname{NGD}(v, b, r, k ; m, n, p$; $\left.\lambda_{1}, \lambda_{2}, \lambda_{3}\right)$ is said to be cyclic, and denoted by $\operatorname{CNGD}\left(v, b, r, k ; m, n, p ; \lambda_{1}, \lambda_{2}, \lambda_{3}\right)$.

In a CNGD design, if $\lambda_{1}=\lambda_{2}$ or $\lambda_{2}=\lambda_{3}$, the design can be reduced to a cyclic $\mathrm{GD}$ design $\operatorname{CGD}\left(v, b, r, k ; p, m n ; \lambda_{1}, \lambda_{3}\right)$ or $\operatorname{CGD}\left(v, b, r, k ; p m, n ; \lambda_{1}, \lambda_{2}\right)$ by combining the first and the second, or the second and the third associate classes, as mentioned in Section 1. Clearly, in a $\operatorname{CGD}\left(m n, b, r, k ; m, n ; \lambda_{1}, \lambda_{2}\right)$ each group must be the subgroup $m Z_{n}=\{0, m, 2 m, \cdots,(n-1) m\}$ of $Z_{m n}$ or its coset (see Mukerjee, Jimbo and Kageyama (1987)). Note that when $\lambda_{1}=\lambda_{2}=\lambda$ in the CGD, it is called a cyclic balanced incomplete block design, denoted by $\mathrm{CB}(k$, $\lambda ; v)$.

For a block $B$ of a $\operatorname{CGD}\left(v, b, r, k ; m, n ; \lambda_{1}, \lambda_{2}\right)$, the orbit of $B$ is defined by $\left\{B+y(\bmod v): y \in Z_{v}\right\}$. The length of an orbit is its cardinality. An orbit of length $v$ is said to be full ; otherwise it is called a short orbit. A base block of an orbit $R$ is any arbitrarily chosen block $B \in R$.

In order to describe our construction, we also need the concept of a difference matrix. Let $D=\left(d_{i j}\right), i=1,2, \cdots, k ; j=1,2, \cdots, g \lambda$, be a matrix with entries from $Z_{g}$ such that the list $\left(d_{i l}-d_{j l}\right)_{l=1, \cdots, g \lambda}$ contains each element of $Z_{g}$ precisely $\lambda$ times for $i \neq j$ and $i, j \in\{1,2, \cdots, k\}$. Then $D$ is called a difference matrix and denoted by $\operatorname{DM}(k, \lambda ; g)$.

THEOREM 4.1. If there exist a $C G D\left(m n, b, r, k ; m, n ; \lambda_{1}, \lambda_{2}\right)\left(\lambda_{1} \leq \lambda_{2}\right)$ without short orbit, and a $D M(k, \lambda ; g)$, then there exists a $C N G D\left(m n g, b_{1}, r_{1}, k ; n, g\right.$, $\left.m ; 0, \lambda_{1} \lambda, \lambda_{2} \lambda\right)$. Furthermore, if there exists a $C G D\left(n g, b^{\prime}, r^{\prime}, k ; n, g ; \lambda_{1}^{\prime}, \lambda_{2}^{\prime}\right)$, then there exists a CNGD (mng, $\left.b_{2}, r_{2}, k ; n, g, m ; \lambda_{1}^{\prime}, \lambda_{2}^{\prime}+\lambda_{1} \lambda, \lambda_{2} \lambda\right)$.

PROOF. For $v=m n$, let the $v g$ treatments be arranged in a rectangular of $v$ rows and $g$ columns as follows :

$$
\begin{array}{cccc}
0 & v & \cdots & (g-1) v \\
1 & v+1 & \cdots & (g-1) v+1 \\
\vdots & \vdots & & \vdots \\
m-1 & v+m-1 & \cdots & (g-1) v+m-1 \\
m & v+m & \cdots & (g-1) v+m \\
\vdots & \vdots & & \vdots \\
2 m-1 & v+2 m-1 & \cdots & (g-1) v+2 m-1 \\
2 m & v+2 m & \cdots & (g-1) v+2 m \\
\vdots & \vdots & & \vdots \\
m n-1 & v+m n-1 & \cdots & (g-1) v+m n-1
\end{array}
$$

Two treatments $x$ and $y$ are first associates if $x-y \in v Z_{g}$; second associ- 
ates if $x-y \in m Z_{n g}-v Z_{g}$; and third associates if $x-y \in Z_{v g}-m Z_{n g}$. This is a nested association scheme with $m$ sets of $n$ groups of $g$ treatments each.

Now let $D=\left(d_{i j}\right)$ be a $\operatorname{DM}(k, \lambda ; g)$. For each base block $B=\left\{b_{1}, \cdots, b_{k}\right\}$ of the $\operatorname{CGD}\left(m n, b, r, k ; m, n ; \lambda_{1}, \lambda_{2}\right)$, construct the following $\lambda g$ base blocks :

$$
B_{j}=\left\{b_{i}+d_{i j} v: i=1,2, \cdots, k\right\}
$$

for $j=1,2, \cdots, \lambda g$. Then every treatment of $v Z_{g}-\{0\}=\{v, \cdots,(g-1) v\}$ does not occur with the treatment 0 , every treatment of $m Z_{n \theta}-v Z_{g}$ occurs together with the treatment 0 exactly $\lambda_{1} \lambda$ times, and every treatment of $Z_{g v}-m Z_{n g}$ occurs together with the treatment 0 exactly $\lambda_{2} \lambda$ times.

Next, for each base block $C=\left\{c_{1}, \cdots, c_{k}\right\}$ of the $\operatorname{CGD}\left(n g, b^{\prime}, r^{\prime}, k ; n, g ; \lambda_{1}^{\prime}\right.$, $\lambda_{2}^{\prime}$ ), let $m C=\left\{m c_{1}, \cdots, m c_{k}\right\}$ be a base block of the desired design. Then any treatment of $m Z_{n g}-v Z_{g}$ occurs together with the treatment $0 \lambda_{2}^{\prime}$ times, and every treatment of $v Z_{g}-\{0\}$ occurs together with the treatment $0 \lambda_{1}^{\prime}$ times. This completes the proof.

Note that the representation of the association scheme defined above is different from that in Section 1.

This theorem generalizes Theorem 1 of Jimbo and Kuriki (1983), Theorem 1 of Jimbo and Vanstone (1984) and Theorem 3.5 of Jimbo (1993). For example, by letting $\lambda_{1}^{\prime}=\lambda \lambda_{2}, \lambda_{2}^{\prime}=\lambda\left(\lambda_{2}-\lambda_{1}\right)$, we obtain a $\operatorname{CB}\left(k, \lambda \lambda_{2} ; v g\right)$, which was obtained firstly by Jimbo (1993)

For more information on cyclic GD designs and difference matrices, see the references mentioned in this section.

\section{NGD designs with $r, k \leq 10$}

A large number of GD designs with $r, k \leq 10$ are available in Clatworthy (1973) and Sinha (1991). Such plans can be used to produce NGD designs through the theorems presented here. For example, a $\mathrm{GD}(4,4,2,2 ; 2,2 ; 0,1)$, SR1, and a GD $(4,8,4,2 ; 2,2 ; 2,1)$, R1, listed in Clatworthy (1973) can produce an $\operatorname{NGD}(8,32,8,2 ; 2,2,2 ; 4,0,1)$ by use of Theorem 3.1 . We list some NGD designs with $r, k \leq 10$ as in Table 1 . Notations $\mathrm{S}, \mathrm{SR}, \mathrm{R}$ in the Source of the table denote GD designs from Clatworthy (1973). Note that a similar table was also listed in Duan and Kageyama (1993). However, they have no overlap between them.

Sinha and Shah (1988) obtained a class of E-optimal 3-concurrence most balanced designs, which may be considered as a generalization of GD designs, to provide a unified approach for identifying certain available classes of GD designs as E-optimal. Sinha and Kageyama (1992) described some methods of constructing E-optimal nested group divisible designs and provided two tables of such designs with $r \leq 20$ and $k \leq 10$. The designs with an asterisk in the present table show such E-optimal designs, four of which are new. Note that the E-optimal design of No. 1 is given in Table 2.1 by Sinha and Kageyama (1992). 
Table 1. NGD designs with $r, k \leq 10$

\begin{tabular}{|c|c|c|c|c|c|c|c|c|c|c|c|c|}
\hline No & $v$ & $r$ & $k$ & $b$ & $m$ & $n$ & $p$ & $\lambda_{1}$ & $\lambda_{2}$ & $\lambda_{3}$ & Source & Remark \\
\hline $1^{*}$ & 8 & 8 & 2 & 32 & 2 & 2 & 2 & 0 & 2 & 1 & SR9, SR2 & Th 2.1 \\
\hline 2 & 8 & 8 & 2 & 32 & 2 & 2 & 2 & 4 & 0 & 1 & SR1, R1 & Th 3.1 \\
\hline 3 & 8 & 9 & 2 & 36 & 2 & 2 & 2 & 1 & 2 & 1 & SR9, R3 & Th 2.1 \\
\hline 4 & 8 & 10 & 2 & 40 & 2 & 2 & 2 & 0 & 1 & 2 & SR10, SR1 & Th 2.1 \\
\hline 5 & 8 & 10 & 2 & 40 & 2 & 2 & 2 & 0 & 3 & 1 & SR9, SR3 & Th 2.1 \\
\hline 6 & 8 & 10 & 2 & 40 & 2 & 2 & 2 & 2 & 0 & 2 & SR1, R3 & Th 3.1 \\
\hline 7 & 8 & 10 & 2 & 40 & 2 & 2 & 2 & 6 & 0 & 1 & SR1, R2 & Th 3.1 \\
\hline 8 & 12 & 6 & 3 & 24 & 2 & 2 & 3 & 0 & 2 & 1 & $\mathrm{~S} 1, \mathrm{R} 54$ & Th 3.1 \\
\hline 9 & 12 & 6 & 3 & 24 & 2 & 2 & 3 & 4 & 0 & 1 & SR18, R42 & Th 3.1 \\
\hline 10 & 12 & 9 & 3 & 36 & 2 & 2 & 3 & 6 & 2 & 1 & R42, R42 & Th 3.1 \\
\hline 11 & 16 & 9 & 3 & 48 & 2 & 2 & 4 & 0 & 3 & 1 & S6, R54 & Th 3.1 \\
\hline 12 & 16 & 9 & 3 & 48 & 2 & 2 & 4 & 6 & 0 & 1 & R54, R42 & Th 3.1 \\
\hline 13 & 18 & 9 & 3 & 54 & 2 & 3 & 3 & 0 & 2 & 1 & R42, SR23 & Th 3.1 \\
\hline 14 & 18 & 9 & 3 & 54 & 3 & 2 & 3 & 6 & 0 & 1 & SR23, R42 & Th 3.1 \\
\hline 15 & 12 & 10 & 3 & 40 & 3 & 2 & 2 & 2 & 3 & 1 & R74, SR18 & Th 2.1 \\
\hline 16 & 18 & 10 & 3 & 60 & 2 & 3 & 3 & 4 & 0 & 1 & SR18, R59 & Th 3.1 \\
\hline 17 & 18 & 10 & 3 & 60 & 3 & 2 & 3 & 0 & 2 & 1 & S30, SR19 & Th 2.1 \\
\hline 18 & 12 & 4 & 4 & 12 & 2 & 2 & 3 & 4 & 0 & 1 & SR18, S1 & Th 3.1 \\
\hline 19 & 12 & 6 & 4 & 18 & 2 & 2 & 3 & 6 & 2 & 1 & S1, S6 & Th 3.1 \\
\hline 20 & 16 & 6 & 4 & 24 & 2 & 2 & 4 & 6 & 0 & 1 & R45, S1 & Th 2.1 \\
\hline $21^{*}$ & 18 & 6 & 4 & 27 & 2 & 3 & 3 & 0 & 2 & 1 & S1, SR41 & Th 3.1 \\
\hline 22 & 18 & 6 & 4 & 27 & 3 & 2 & 3 & 6 & 0 & 1 & SR23, S1 & Th 3.1 \\
\hline 23 & 12 & 8 & 4 & 24 & 2 & 2 & 3 & 0 & 4 & 2 & S1, SR36 & Th 3.1 \\
\hline 24 & 12 & 8 & 4 & 24 & 2 & 2 & 3 & 8 & 0 & 2 & SR18, S2 & Th 3.1 \\
\hline 25 & 12 & 8 & 4 & 24 & 2 & 3 & 2 & 6 & 0 & 2 & SR1, R94 & Th 3.1 \\
\hline 26 & 18 & 8 & 4 & 36 & 2 & 3 & 3 & 6 & 0 & 1 & SR18, R104 & Th 3.1 \\
\hline 27 & 18 & 8 & 4 & 36 & 3 & 2 & 3 & 4 & 2 & 1 & S21, R109 & Th 3.1 \\
\hline $28^{*}$ & 24 & 8 & 4 & 48 & 2 & 4 & 3 & 0 & 2 & 1 & S1, SR44 & Th 3.1 \\
\hline 29 & 24 & 8 & 4 & 48 & 4 & 2 & 3 & 8 & 0 & 1 & SR26, S1 & Th 3.1 \\
\hline 30 & 24 & 9 & 4 & 54 & 2 & 3 & 4 & 0 & 3 & 1 & S6, SR41 & Th 3.1 \\
\hline 31 & 24 & 9 & 4 & 54 & 3 & 2 & 4 & 9 & 0 & 1 & SR41, S6 & Th 3.1 \\
\hline $32^{*}$ & 8 & 10 & 4 & 20 & 2 & 2 & 2 & 2 & 4 & 3 & SR38, SR1 & Th 2.7 \\
\hline 33 & 12 & 10 & 4 & 30 & 2 & 2 & 3 & 6 & 4 & 2 & S1, R97 & Th 3.1 \\
\hline 34 & 18 & 10 & 4 & 45 & 3 & 2 & 3 & 10 & 2 & 1 & S21, S11 & Th 3.1 \\
\hline 35 & 24 & 10 & 4 & 60 & 2 & 2 & 6 & 10 & 0 & 1 & $\mathrm{R} 70, \mathrm{Sl}$ & Th 3.1 \\
\hline $36^{*}$ & 30 & 10 & 4 & 75 & 2 & 5 & 3 & 0 & 2 & 1 & S1, SR46 & Th 3.1 \\
\hline 37 & 30 & 10 & 4 & 75 & 5 & 2 & 3 & 10 & 0 & 1 & SR28, S1 & Th 3.1 \\
\hline 38 & 12 & 10 & 5 & 24 & 2 & 2 & 3 & 4 & 6 & 3 & S1, R134 & Th 3.1 \\
\hline 39 & 16 & 10 & 5 & 32 & 2 & 4 & 2 & 8 & 0 & 2 & SR1, R133 & Th 3.1 \\
\hline 40 & 18 & 10 & 5 & 36 & 2 & 3 & 3 & 2 & 4 & 2 & S1, R145 & Th 3.1 \\
\hline 41 & 18 & 10 & 5 & 36 & 2 & 3 & 3 & 8 & 0 & 2 & SR18, R137 & Th 3.1 \\
\hline 42 & 18 & 10 & 5 & 36 & 3 & 2 & 3 & 0 & 4 & 2 & S21, R144 & Th 3.1 \\
\hline 43 & 24 & 10 & 5 & 48 & 2 & 4 & 3 & 8 & 0 & 1 & SR18, R143 & Th 3.1 \\
\hline 44 & 36 & 10 & 5 & 72 & 3 & 4 & 3 & 0 & 2 & 1 & S21, R153 & Th 3.1 \\
\hline 45 & 18 & 4 & 6 & 12 & 2 & 3 & 3 & 4 & 0 & 1 & SR18, S21 & Th 3.1 \\
\hline 46 & 12 & 6 & 6 & 12 & 2 & 2 & 3 & 6 & 4 & 2 & S1, S18 & Th 3.1 \\
\hline 47 & 18 & 6 & 6 & 18 & 2 & 3 & 3 & 6 & 2 & 1 & $\mathrm{~S} 1, \mathrm{~S} 27$ & Th 3.1 \\
\hline 48 & 24 & 6 & 6 & 24 & 2 & 3 & 4 & 6 & 0 & 1 & R54, S21 & Th 3.1 \\
\hline 49 & 27 & 6 & 6 & 27 & 3 & 3 & 3 & 6 & 0 & 1 & SR23, S21 & Th 3.1 \\
\hline 50 & 18 & 8 & 6 & 24 & 2 & 3 & 3 & 8 & 0 & 2 & SR18, S22 & Th 3.1 \\
\hline
\end{tabular}


Table 1. (continued)

\begin{tabular}{|c|c|c|c|c|c|c|c|c|c|c|c|c|}
\hline No & $v$ & $r$ & $k$ & $b$ & $m$ & $n$ & $p$ & $\lambda_{1}$ & $\lambda_{2}$ & $\lambda_{3}$ & Source & Remark \\
\hline 51 & 18 & 8 & 6 & 24 & 3 & 2 & 3 & 0 & 4 & 2 & S21, SR66 & Th 3.1 \\
\hline 52 & 36 & 8 & 6 & 48 & 4 & 3 & 3 & 8 & 0 & 1 & SR26, S21 & Th 3.1 \\
\hline 53 & 16 & 9 & 6 & 24 & 2 & 2 & 4 & 9 & 6 & 2 & S6, S18 & Th 3.1 \\
\hline 54 & 24 & 9 & 6 & 36 & 2 & 3 & 4 & 9 & 3 & 1 & S6, S27 & Th 3.1 \\
\hline 55 & 24 & 9 & 6 & 36 & 3 & 2 & 4 & 9 & 0 & 2 & SR41, S18 & Th 3.1 \\
\hline 56 & 36 & 9 & 6 & 54 & 3 & 3 & 4 & 9 & 0 & 1 & SR41, S27 & Th 3.1 \\
\hline 57 & 18 & 10 & 6 & 30 & 3 & 2 & 3 & 10 & 4 & 2 & S21, S28 & Th 3.1 \\
\hline 58 & 27 & 10 & 6 & 45 & 3 & 3 & 3 & 10 & 2 & 1 & S21, S38 & Th 3.1 \\
\hline 59 & 36 & 10 & 6 & 60 & 2 & 3 & 6 & 10 & 0 & 1 & R70, S21 & Th 3.1 \\
\hline 60 & 45 & 10 & 6 & 75 & 3 & 5 & 3 & 0 & 2 & 1 & S21, SR75 & Th 3.1 \\
\hline 61 & 45 & 10 & 6 & 75 & 5 & 3 & 3 & 10 & 0 & 1 & SR28, S21 & Th 3.1 \\
\hline 62 & 24 & 4 & 8 & 12 & 2 & 4 & 3 & 4 & 0 & 1 & SR18, S53 & Th 3.1 \\
\hline 63 & 24 & 6 & 8 & 18 & 2 & 4 & 3 & 6 & 2 & 1 & S1, S61 & Th 3.1 \\
\hline 64 & 32 & 6 & 8 & 24 & 2 & 4 & 4 & 6 & 0 & 1 & R54, S53 & Th 3.1 \\
\hline 65 & 36 & 6 & 8 & 27 & 3 & 4 & 3 & 6 & 0 & 1 & SR23, S53 & Th 3.1 \\
\hline 66 & 24 & 8 & 8 & 24 & 2 & 4 & 3 & 8 & 0 & 2 & SR18, S54 & Th 3.1 \\
\hline 67 & 48 & 8 & 8 & 48 & 4 & 4 & 3 & 8 & 0 & 1 & SR26, S53 & Th 3.1 \\
\hline 68 & 32 & 9 & 8 & 36 & 2 & 4 & 4 & 9 & 3 & 1 & S6, S61 & Th 3.1 \\
\hline 69 & 48 & 9 & 8 & 54 & 3 & 4 & 4 & 9 & 0 & 1 & SR41, S61 & Th 3.1 \\
\hline 70 & 36 & 10 & 8 & 45 & 3 & 4 & 3 & 10 & 2 & 1 & S21, S69 & Th 3.1 \\
\hline 71 & 48 & 10 & 8 & 60 & 2 & 4 & 6 & 10 & 0 & 1 & R70, S53 & Th 3.1 \\
\hline 72 & 60 & 10 & 8 & 75 & 5 & 4 & 3 & 10 & 0 & 1 & SR28, S53 & Th 3.1 \\
\hline 73 & 18 & 6 & 9 & 12 & 2 & 3 & 3 & 6 & 4 & 2 & S1, S82 & Th 3.1 \\
\hline 74 & 24 & 9 & 9 & 24 & 2 & 3 & 4 & 9 & 6 & 2 & S6, S18 & Th 3.1 \\
\hline 75 & 36 & 9 & 9 & 36 & 3 & 3 & 4 & 9 & 0 & 2 & SR41, S82 & Th 3.1 \\
\hline 76 & 27 & 10 & 9 & 30 & 3 & 3 & 3 & 10 & 4 & 2 & S21, S86 & Th 3.1 \\
\hline 77 & 30 & 4 & 10 & 12 & 2 & 5 & 3 & 4 & 0 & 1 & SR18, S100 & Th 3.1 \\
\hline 78 & 30 & 6 & 10 & 18 & 2 & 5 & 3 & 6 & 2 & 1 & S1, S106 & Th 3.1 \\
\hline 79 & 40 & 6 & 10 & 24 & 2 & 5 & 4 & 6 & 0 & 1 & R54, S100 & Th 3.1 \\
\hline 80 & 45 & 6 & 10 & 27 & 3 & 5 & 3 & 6 & 0 & 1 & SR23, S100 & Th 3.1 \\
\hline 81 & 30 & 8 & 10 & 24 & 2 & 5 & 3 & 8 & 0 & 2 & SR18, S101 & Th 3.1 \\
\hline 82 & 60 & 8 & 10 & 48 & 4 & 5 & 3 & 8 & 0 & 1 & SR26, S100 & Th 3.1 \\
\hline 83 & 40 & 9 & 10 & 36 & 2 & 5 & 4 & 9 & 3 & 1 & S6, S106 & Th 3.1 \\
\hline 84 & 60 & 9 & 10 & 54 & 3 & 5 & 4 & 9 & 0 & 1 & SR41, S106 & Th 3.1 \\
\hline 85 & 45 & 10 & 10 & 45 & 3 & 5 & 3 & 10 & 2 & 1 & S21, S114 & Th 3.1 \\
\hline 86 & 60 & 10 & 10 & 60 & 2 & 5 & 6 & 10 & 0 & 1 & R70, S100 & Th 3.1 \\
\hline 87 & 75 & 10 & 10 & 75 & 5 & 5 & 3 & 10 & 0 & 1 & SR28, S100 & Th 3.1 \\
\hline
\end{tabular}

\section{Acknowledgements}

The research was supported in part by a Grant-in-Aid for Co-operative Research (A) 07304021 from the Japanese Ministry of Education, and by Research Fellowships from the Japan Society for the Promotion of Science for Young Scientists. The authors thank the referees for their helpful comments.

\section{REFERENCES}

[1] Bhagwandas, Sinha, K. and Kageyama, S. (1992). Constructions of PBIB designs based on nested group divisible association scheme, Utilitas Math., 41, 169-174. 
[2] Clatworthy, W. H. (1973). Tables of Two-Associate-Class Partially Balanced Designs, NBS Appl. Math., Ser. 63, Washington, D. C., USA.

[3] Duan, X. and Kageyama, S. (1993). Constructions of nested group divisible designs, Statist. \& Prob. Lett., 18, 41-48.

[4] Duan, X. and Kageyama, S. (1995). Constructions of group divisible designs, J. Japan Statist. Soc., 25(2), 143-150.

[5] Hinkelmann, K. and Kempthorne, O. (1963). Two classes of group divisible partial diallel crosses, Biometrika, 50, 281-291.

[6] Jimbo, M. (1993). Recursive constructions for cyclic BIB designs and their generalizations, Discrete Math., 116, 79-95.

[ 7 ] Jimbo, M. and Kuriki, S. (1983). On a composition of cyclic 2-designs, Discrete Math., 46, 249-255.

[8] Jimbo, M. and Vanstone, S. (1984). Recursive constructions for resolvable and doubly resolvable 1-rotational Steiner 2.designs, Utilitas Math., 26, 45-61.

[ 9 ] Kageyama, S. and Tanaka, T. (1981). Some families of group divisible designs, J. Statist. Plann. Inference, 5, 231-241.

[10] Mukerjee, R., Jimbo, M. and Kageyama, S. (1987). On cyclic semi-regular group divisible designs, Osaka J. Math., 24, 395-407.

[11] Raghavarao, D. (1960). A generalization of group divisible designs, Ann. Math. Statist., 31, 756-771.

[12] Roy, P. M. (1953). Hierarchical group divisible incomplete block designs with $m$ associate classes, Science and Culture, 19, 210-211.

[13] Roy, P. M. (1962). On the properties and construction of HGD designs with $m$ associate classes, Calcutta Statist. Assoc. Bull., 11, 10-38.

[14] Sinha, B. K. and Shah, K. R. (1988). Optimality aspects of 3-concurrence most balanced designs, J. Statist. Plann. Inference, 20, 229-236.

[15] Sinha, K. (1991). A list of new group divisible designs, J. Res. Natl. Inst. Stand. Technol., 96, 613-615.

[16] Sinha, K. and Kageyama, S. (1992). Constructions of some E-optimal 3-concurrence most balanced designs, J. Statist. Plann. Inference, 31, 127-132. 